\title{
Apprentissage immersif du FLE: potentiels des applications et scénario ludo-éducatif
}

\section{Immersive learning of French as a foreign language: potential of applications and edutainment scenario}

\author{
Claude Duée \\ Universidad Castilla-La Mancha \\ claude.duee@uclm.es \\ Justine Martin \\ Universidad de Castilla-La Mancha \\ Justine.Martin@uclm.es
}

\begin{abstract}
Our research group, LCF, is interested in addressing the topic of virtual reality under its different forms, such as video games, augmented reality, in the teaching of a foreign language, thinking that it could be the solution for our students. Indeed, they can approach, in our case, French, from gamification and, therefore, from motivation. In addition, it has the advantage of being able to take learning to the field of autonomy and, above all, of immersion, a concept that seems essential to achieve a language acquisition and that we have to define in relation to our objective. Afterwards, our research has continued its course looking for the different interactive applications and analyzing them to observe to what extent they bring a benefit for this learning and, if not, what solution we can give it.
\end{abstract}

\section{Keywords}

immersion, video games, virtual reality, augmented reality, interactive videos.

\begin{abstract}
Resumen
Nuestro grupo de investigación, LCF, está interesado en abordar la temática de la realidad virtual bajos sus diferentes formas, como los videojuegos, la realidad aumentada, en la enseñanza de una lengua extranjera, pensando que podría ser la solución para nuestros estudiantes. En efecto, pueden abordar, en nuestro caso, el francés, desde la gamificación y, por tanto, desde la motivación. Además, tiene la ventaja de poder llevar el aprendizaje al terreno de la autonomía y, sobre todo, de la inmersión, concepto que nos parece primordial para alcanzar una adquisición de una lengua y que tenemos que definir en relación con nuestro objetivo. Después, nuestra investigación ha seguido su curso buscando las diferentes aplicaciones interactivas y analizándolas para observar en qué medida conllevan un beneficio para este aprendizaje $y$, en caso de que no fuera así, qué solución le podemos dar.
\end{abstract}

\section{Palabras claves}

inmersión, videojuegos, realidad virtual, realidad aumentada, videos interactivos. 


\section{Introduction}

Nous entendons souvent parler de serious game, des jeux réalisés pour un objectif d'apprentissage concret. Mais il existe également le serious gaming qui est lui un jeu que l'on a détourné de son objectif premier pour l'élever sur l'autel de l'apprentissage. En ce qui concerne le serious gaming, il faut avancer qu'il s'agit, dans les langues étrangères, de quelque chose qui n'est pas nouveau. Ce qui l'est, ce sont les supports: réalité virtuelle, jeux vidéo, vidéos interactives. Et en tout cas, utiliser ces systèmes, c'est tenter d'immerger l'apprenant dans un monde virtuel dans lequel il apprendrait, par exemple, le français d'une manière plus rapide et plus durable. Mais que veut dire immersion?

Le terme immersion signifie, selon le CNRTL, "le fait de plonger ou être plongé dans une certaine ambiance", le fait de "se fondre dans quelque chose, ou quelqu'un." On connaît d'ailleurs tous l'immersion linguistique où l'on se rend dans le pays de la langue en question afin de s'immerger dans sa culture. L'apprentissage immersif (AI, ou Immersive Learning en anglais) grâce à des technologies virtuelles, surtout développées en entreprise, réside dans l'immersion de l'apprenant non pas dans le pays de la langue que l'on désire apprendre, mais dans un univers virtuel qui nous offre la possibilité de vivre une expérience qui le plonge dans une autre réalité, coupé de l'environnement dans lequel il se trouve, et à laquelle il "croit", sans avoir à se déplacer.

Plus techniquement, selon les créateurs de Videolearning, "l'apprentissage immersif fait appel à la conception d'environnement créé à partir d'images de synthèse en 3 dimensions, dans lequel l'apprenant est plongé d'une manière immersive (lunettes, casques, et écrans) et proposant un espace de simulation idéal pour la formation." L'AI se réalise surtout grâce aux technologies telles que la RV (réalité virtuelle) ou encore celle de la RA (réalité augmentée) où un univers réel est augmenté, enrichi d'éléments virtuels. Le jeu vidéo, autre technologie qui permet une immersion de l'utilisateur, place, selon Schmoll (2017), "le joueur-apprenant dans une position active. Il est, de ce fait, le lieu d'un apprentissage constructiviste: parce qu'il est mis en situation, immergé dans l'univers virtuel, le joueur est amené à structurer son savoir par l'observation, l'action, l'expérimentation" (16). Selon cette auteure, cet apprentissage immersif peut donc se faire aussi à travers le jeu vidéo ou les vidéos interactives: on parle alors d'immersion fictionnelle, à travers un univers totalement virtuel. Mais comment se traduit l'immersion à travers la réalité virtuelle dans l'apprentissage d'une langue étrangère?

\section{L'immersion en question}

L'immersion consiste en un processus dans lequel le joueur ou l'utilisateur va se laisser "prendre au jeu”. Georges (2013) explique que "l'expérience immersive est déterminée par l'engagement actif du joueur dans l'expérience ludique et par son consentement à une 
certaine forme de dépossession de soi" (51). L'immersion devrait donc favoriser la présence et elle est en lien direct avec la disposition du joueur ou de l'utilisateur à répondre à l'environnement virtuel/fictionnel comme s'il s'agissait de la vie réelle.

Selon Arsenault et Picard (2008), l'immersion se divise en trois types: l'immersion sensorielle, l'immersion basée sur le défi et l'immersion fictionnelle. L'immersion sensorielle, comme son nom l'indique, permet au joueur, grâce aux sens, de se concentrer totalement sur le monde du jeu en question et ses stimuli. L'immersion basée sur le défi est "spécifique au jeu vidéo et se produit lorsqu'un point d'équilibre est atteint entre les habiletés du joueur et les défis proposés par le jeu" (3). Plus le joueur contrôlera les règles, plus il aura de facilité pour s'immerger. L'immersion fictionnelle est le phénomène qui permet au joueur d'être absorbé par l'histoire, par le monde diégétique du jeu. Grâce à ce processus, il va s'identifier ou s'attacher au personnage. Effectivement, Genvo explique, dans son introduction au dossier spécial de la revue Science du jeu (2018) que:

[...] les jeux se rapprochent notamment de la narration par l'intermédiaire de la fiction, en ce que les narrations littéraires ou cinématographiques de fiction reposent sur le fait de faire-croire, tout comme peuvent le faire les jeux vidéo. (3)

Cette immersion fictionnelle est sous-catégorisée en trois types d'immersion: diégétique, narrative et identificatrice. L'immersion diégétique "renvoie à l'impression d'être présent dans l'univers fictionnel d'un jeu et serait influencée par la qualité et la quantité des détails fournis dans cet espace" (Trépanier-Jobin \& Couturier, 2018: 12). On parle ici de sensation de présence dans le monde virtuel, d'exploration spatiale. L'immersion narrative renvoie au développement du récit, de l'histoire. Elle est liée à l'aspect temporel et "aux stratégies narratives mises en place dans le jeu et au désir qu'a le joueur de faire progresser l'histoire" (ibid.). Finalement, l'immersion identificatrice renvoie, quant à elle, aux émotions et survient lorsqu'il se créé un lien entre le personnage et le joueur.

Une fois qu'ont été dégagés et expliqués les trois grands types d'immersion et leurs sous catégories, il faut souligner que lorsqu'un joueur joue, l'immersion peut se produire à différents niveaux. Il y a donc trois différents degrés d'immersion:

- L'engagement, premier palier de l'immersion. Selon Arsenault et Picard (2008), est nécessaire un certain investissement de la part du joueur et une bonne accessibilité et intelligibilité de la part du jeu (intuitif). Une fois engagé dans le jeu, le joueur continuera à jouer.

- L'absorption, deuxième palier, autrement dit l'investissement émotionnel du joueur qui se définit en fonction du graphisme et des tâches à accomplir.

- L'immersion totale, troisième palier, qui reprend les deux types d'immersions déjà citées auparavant. 


\section{Apprentissage immersif des langues par technologies virtuelles et la perspective actionnelle}

Dans son article sur l'intégration du jeu vidéo en classe de langue, Schmoll (2017) propose de mettre en relation le jeu vidéo et la perspective actionnelle à travers un schéma très explicite:

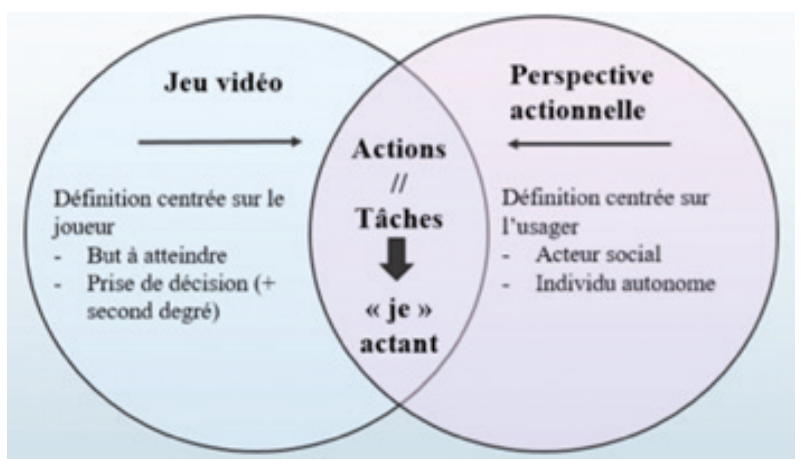

Figure 1: Lien entre le jeu vidéo et perspective actionnelle de Schmoll (2017).

Selon cette auteure, le jeu vidéo, et donc l'équivalent de n'importe quelle technologie virtuelle permettant l'AI, entrent parfaitement en osmose avec les principes défendus par la perspective actionnelle dans la mesure où l'apprenant doit réaliser une action ou une tâche concrète en prenant des décisions. Le jeu vidéo, par exemple, comme la perspective actionnelle, se centre sur le sujet jouant, soit le "je actant" qui prend une position active face à son apprentissage (toutes les décisions prises auront des conséquences).

Nous avons ici souhaité reprendre son schéma et l'adapter à tous types de technologies virtuelles d'apprentissage immersif:

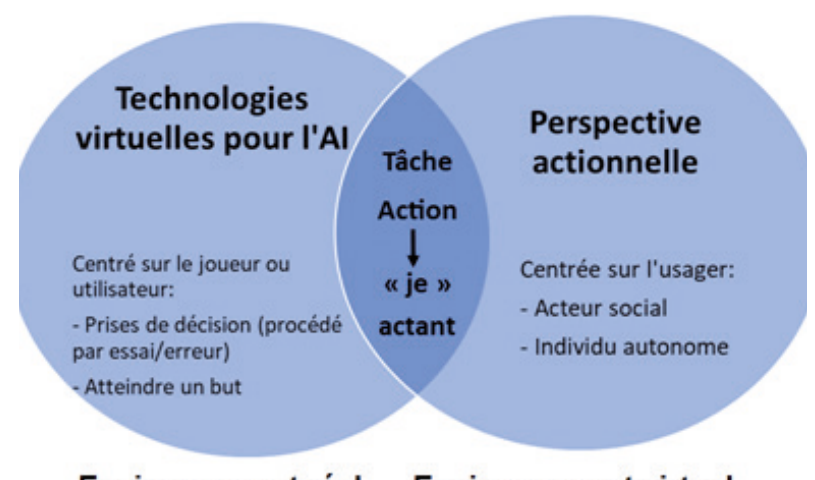

\section{Environnement réel Environnement virtuel}

Figure 2: Lien entre les technologies virtuelles pour l'apprentissage immersif et perspective actionnelle. 
Les utilisateurs/usagers vont être introduits dans un univers graphique qui va contextualiser la ou les missions à accomplir, la ou les buts à atteindre. Ils vont agir à travers leur personnage ou avatar dans des situations différentes et devront prendre des décisions à partir de l'observation, puis l'action en partant de leurs connaissances en langue cible. Ces prises de décision les amèneront à avancer par essai/erreur.

À défaut de pouvoir proposer des tâches réelles, ces technologies deviennent une alternative parfaite pour l'enseignant. Mais, pour pouvoir remédier à ces tâches virtuelles, le scénario pédagogique est indispensable. En effet, proposer ces outils en classe de langue, c'est proposer "une activité de second degré" (Schmoll, 2017:13) qui permettra à l'apprenant de s'isoler momentanément "du cadre primaire", c'est-à-dire le contexte de classe. Le scénario pédagogique sert alors à délimiter les possibilités qui s'offrent ou pourraient s'offrir à l'utilisateur de l'AI et les solutions en cas d'obstacles ou de problèmes. Enfin, c'est l'assurance que les objectifs de départ et les résultats recherchés ne sont pas à la merci de variantes incontrôlables. Dans cette perspective, et avec ces prémices, l'immersion ludique, sensorielle et visuelle permettraient "un apprentissage dans lequel l'apprenant peut expérimenter sans danger" (Schmoll, 2017: 14).

En ce qui concerne le rapport direct entre l'immersion et l'apprentissage, dans leur article, Noeppel et al. (2013) expliquent que "le niveau d'immersion a une influence significative sur l'apprentissage, une plus grande immersion entraînant un meilleur apprentissage de contenus disciplinaires avec [...] une meilleure compréhension des processus" (33-34).

\section{Principaux systèmes immersifs pour l'apprentissage d'une langue étrangère}

Dans un article de Ouramdane, Otmane et Mallem, de 2009, Interaction 3D en Réalité Virtuelle. État de l'art, ces chercheurs reprenaient le concept unificateur de Paul Milgram (Milgram et al., 1994) qui établissait un continuum partant du réel pour arriver au virtuel. Nous reprenons le schéma qu'ils ont adapté à partir de celui de Milgram:

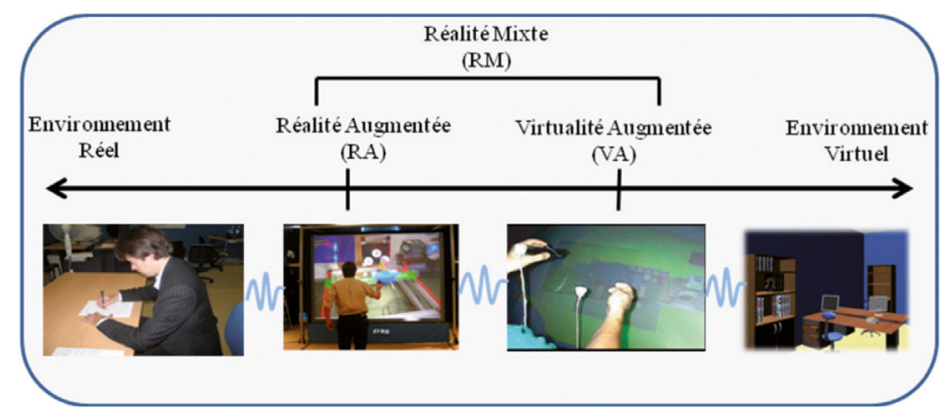

Figure 3: Continuum partant du réel pour arriver au virtuel de Ouramdane, Otmane et Mallem (2009). 
Ce tableau nous montre que les différents systèmes semblent éloigner l'environnement réel et l'environnement virtuel. Et c'est normal car il s'agit d'une approche technique, objective. Or, pour nous, professeur de langue étrangère, la technique est au service de quelque chose de bien plus important, l'apprenant. C'est lui qui est au centre du débat, c'est lui qui a besoin d'apprendre. Donc le défi, c'est justement de rapprocher ces deux espaces mais du point de vue de l'utilisateur et de son expérience comme étant à la fois apprenant et apprenant immergé. Nous reprenons donc la définition qui nous semble s'adapter à nos besoins de RV, celle de Fuchs et al. (2003: 5) qui pose que "la finalité de la réalité virtuelle est de permettre à une personne (ou plusieurs) une activité sensori-motrice et cognitive dans un monde artificiel, créé numériquement, qui peut être imaginaire, symbolique ou une simulation de certains aspects du monde réel'. Il s'agit donc bien de trois items qui se trouvent à la base de toute RV, "l'immersion, l'interaction et l'imagination".

\subsection{La réalité virtuelle}

Selon Lewis, Plante et Lemire (2021), en 1968 déjà, Sutherland, de l'Université d'Harvard (USA), a développé un dispositif virtuel utilisant un casque qui permettait à l'utilisateur d'entrer dans un monde créé par un ordinateur. L'utilisateur ne voit plus la réalité, comme dans le fameux film Matrix. La réalité virtuelle, aujourd'hui, est un hyperonyme qui englobe des applications comme Mondly où il s'agit de mettre en place un espace, des personnages, que l'on sait faux, mais auxquels on croit, comme une réceptionniste d'un hôtel (figure 4, ci-dessous, image de droite). Dans cette figure 4, on peut apprécier le maniement de cette réalité augmentée:

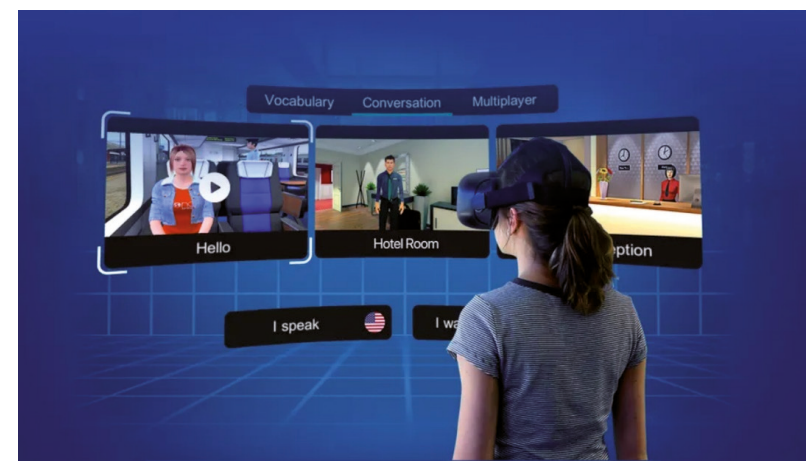

Figure 4, Mondly AR.

D'une part, on peut voir qu'il y a une interaction, ne serait-ce qu'avec le choix de la langue. Il faut pourtant préciser ce que signifie interaction. En effet, il faut comprendre ce terme non pas comme une communication naturelle, un échange de paroles, où les ratés 
ne sont pas rares, mais plutôt une intervention gestuelle, comme appuyer sur une ou l'autre parole écrite dans un encadré pour dire, pour faire, et où les ratés n'existent pas.

D'autre part, interaction signifie immersion, car, quand on communique, le monde qui nous entoure n'existe plus. En outre, dans le cas de la figure 4 (ci-dessus), on peut penser que cette interaction non naturelle est un frein à une immersion, mais il y a le casque qui fait entrer le sujet actant dans le monde virtuel, éloigné de son réel, ce qui rendrait l'apprentissage bien plus motivant et efficace. À l'opposé, la figure 5 (ci-dessous), offre cette même application sans que l'on soit plongé dans la $A R$, c'est-à-dire sans le casque, le sujet apprenant restant en contact avec la réalité du monde qui l'entoure. Ce qui nous amène à poser la question de savoir si c'est le système (dans ce cas-ci le casque et l'application), comme le proposait Milgram et al. (1994) qui donne tout son sens à l'immersion ou s'il s'agit d'autre chose. En ce qui nous concerne, nous pensons que c'est la personne et son degré d'hypnotisation qui rend la sensation d'immersion plus ou moins intense. Plus exactement, il s'agit du ressenti de l'apprenant et non de la description objective, c'est-à-dire la caractéristique de la machine ou de l'objet (casque ou App) qui rend possible l'immersion.

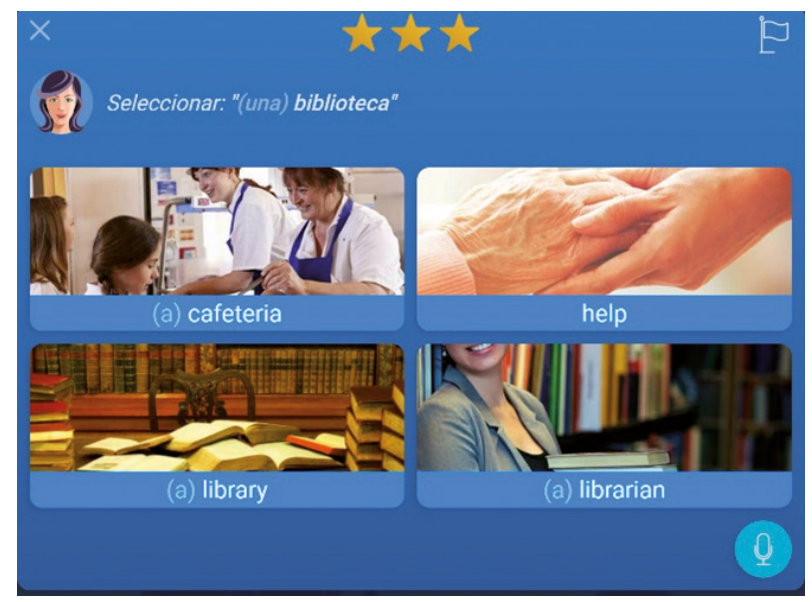

Figure 5: Mondly.

En effet, dans cette application (figure 5) apparue avant Mondly AR, il n'y absolument aucune coupure avec la réalité du sujet. Il ne resterait donc des trois items applicables à cette dernière, c'est-à-dire, immersion, interaction et imagination, que les deux dernières. Il faut donc, à notre avis, que l'imagination soit très active pour rendre l'apprentissage ludique et donc que l'engagement ait lieu, ou que le sujet apprenant soit hypnotisé par l'application. En tout cas, il est clair que la réalité virtuelle de la figure 4 et 5 relève de l'immersion sensorielle, et cette dernière (la figure 5) se rapproche aussi de l'immersion fictionnelle, même si elle est loin d'être celle de la réalité augmentée. En effet, Mondly peut provoquer l'engagement du sujet actant, Mondly AR l'absorption, mais ni l'une ni l'autre n'entraîne l'immersion totale. 
Si l'on prend l'exemple de la réceptionniste de l'hôtel, l'apprenant pourra choisir des actes de paroles qui donneront lieu, à leur tour, à un choix de réponses offertes au sujet. Dans la version AR, la seule différence, c'est une sensation d'immersion plus forte, mais les choix et les interactions avec l'objet seront les mêmes. Voyons ce qu'il en est pour la réalité augmentée.

\subsection{La réalité augmentée}

Né au début des années 1990, les termes réalité augmentée désignent une forme d'interaction utilisateur-machine, "basée sur l'association d'objets réels, issus de l'environnement de l'utilisateur, et virtuels, c'est-à-dire créés par l'ordinateur' Dugas, 2016: 3. Dans ce cas-ci, la RA n'isole pas l'utilisateur dans le monde virtuel. Il a aussi prise sur la réalité. Ensuite, il existe la réalité augmentée étendue, que nous verrons plus tard, qui permet à l'utilisateur de pouvoir contrôler, changer, transformer cette réalité virtuelle. Donc, l'adjectif augmenté indique que l'on parle d'un système qui fusionne trois éléments: premièrement, le mixte du monde virtuel et du monde réel, deuxièmement, l'interaction avec des objets, et troisièmement la possibilité de manipulation de ces objets grâce aux images 3D (Marin Díaz y Sampedro-Requena, 2020). Cela élève le niveau d'immersion à un niveau supérieur à celui vu précédemment, d'autant plus que le monde virtuel en question non seulement est manipulable mais aussi identique (ou presque) aux objets quotidiens (Lewis et al., 2021: 14).

Une expérience de cette sorte ne manquera pas de susciter l'intérêt et donc la motivation pour un apprenant de LE. En effet, faire bouger des objets qui ressemblent aux objets réels mais que l'on sait virtuels est, d'une part, quelque chose qui sort du quotidien et, d'autre part, cela donne une prise, un contrôle ou un semblant de contrôle sur ces objets et élève la sensation d'immersion qui est de plus en plus proche de l'immersion totale. La seule différence, c'est l'aspect danger qui n'existe pas car il n'est pas ressenti. Intervenir chirurgicalement un malade, être (virtuellement) dans la salle d'opération sans courir le risque de faire une mauvaise manipulation qui mettrait en danger le patient est rassurant. Le sujet-apprenant n'a aucun mal à s'engager, à être motivé, car s'il rate quelque chose, c'est du faux, il peut recommencer. Prenons l'exemple d'une scène de niveau B2, où un sujet-apprenant de langue étrangère se retrouve en face d'une situation injuste, comme une femme maltraitée. Il doit prendre position et choisir entre continuer son chemin, prendre le bâton qui se trouve dans un coin de la scène ou appeler la police à l'aide du téléphone qu'il tient dans les mains. Peu importe ce qu'il décidera. Ce sont ces choix et prises de décision qui feront avancer l'histoire et qui donneront lieu à la phase d'argumentation (niveau B2) postérieure que l'on peut espérer plus riche puisque le sujet-actant se sera immergé complètement dans cette réalité.

La différence saute aux yeux si l'on compare la RA avec une application comme Mondly ou Mondly AR. Ces dernières applications ne peuvent dépasser le niveau B1, niveau 
qui ne requiert pas les subtilités d'un niveau B2, et encore moins d'un C1-C2. En effet, ces applications, pour l'instant, sont faites pour un usage quotidien qui est celui du A1 jusqu'au B1 au grand maximum. La réalité augmentée, on vient de le voir, peut être une aide précieuse pour un niveau B2. La réalité mixte sera, à notre avis, encore beaucoup plus apte à s'adapter à un niveau $\mathrm{B} 2$ et même $\mathrm{C} 1$. C'est ce que nous allons voir ci-après.

\subsection{La réalité mixte}

La réalité mixte combine la réalité virtuelle et la réalité augmentée. Un utilisateur peut communiquer avec un autre personnage virtuel, ajouter des éléments virtuels à la réalité, au monde réel, et ainsi parler "comme si l'on était l'un en face de l'autre réellement" et bientôt, nous dit-on, les sensations, les sentiments vont pouvoir être "sentis". Ainsi, je vais pouvoir réellement mais aussi virtuellement embrasser mon frère qui habite à $2500 \mathrm{~km}$ et je devrais pouvoir avoir la sensation de l'embrasser.

Pour l'instant, aucune expérience, à notre connaissance, n'a été mise en place à l'aide de la RM en classe de langue étrangère. En tout cas, en médecine, par exemple, l'utilisation de cette RM pour un futur chirurgien paraît indispensable. Mais on peut penser dans un avenir (proche) à son utilisation, par exemple, pour apprendre le vocabulaire des plantes, à aller chercher des champignons dans le bois avec un panier et ensuite cuisiner pour des amis ces champignons. L'apprenant serait réellement immergé et les sensations seraient vraies, donc, à notre avis, l'apprentissage serait optimum pour un B2 et $\mathrm{C} 1$.

\section{Problèmes avec la RV pour l'apprentissage}

Que peut-on faire avec ces réalités virtuelles plus ou moins immersives en cours de langue étrangère? Il semblerait que n'importe quelle expérience pourrait-être assez surprenante et susciter l'intérêt des apprenants. Mais tout d'abord, le premier obstacle sera la manipulation correcte du support et l'entrée dans ce monde virtuel pour un apprenant. Le deuxième obstacle repose sur les caractéristiques de ces casques qui ne peuvent être utilisés à certains âges et très longtemps. Le troisième obstacle est l'autonomie de l'apprenant. En effet, la personne doit plus ou moins aimer ce genre d'objets et, ainsi, avoir la présence d'esprit de s'en servir chez elle car en classe, son utilisation est limitée. Quatrième obstacle: le risque de lassitude. Cinquième obstacle: peut-on utiliser ces RV à tous les niveaux? Nous ne le croyons pas. Par conséquent, la compétence linguistique est limitée par les niveaux, par le temps mis pour apprendre à manipuler l'objet en question. Cela dit, un apprenant qui découvre à la fois la RV à travers une application et la langue ne peut que le séduire. D'abord, nous le disions ci-dessus, c'est la nouveauté. En plus, une langue à ses débuts est assez facile à apprendre si les langues sont de la même famille: l'espagnol pour 
un Français ou un Italien. Mais, comme avec les méthodes, il existe le risque de tomber dans la routine, donc, faisons en sorte que le début soit prometteur, et que la suite puisse combler les espoirs de maîtriser la langue cible.

Ces réflexions qui sont les écueils de la RV en langues étrangères ne prouvent évidemment pas que celle-ci ne fonctionne pas. Mais, si l'immersion dans un monde autre que le mien n'est possible qu'avec un objet comme le casque ou les lunettes qui nous obligent à nous écarter de ce que nous connaissons, à oublier le réel, et qui, à priori, serait le moyen d'optimiser la motivation, on peut à juste titre se demander, d'une part, si cela va vraiment et durablement être le moteur d'un apprentissage d'une LE, et d'autre part, de quelle manière faut-il s'y prendre. N'est-ce pas la découverte en elle-même qui semble attirante, justement car c'est nouveau? Est-ce que la RV, comme hyperonyme, va vraiment faciliter l'engagement, l'absorption et donc l'apprentissage? N'est-ce pas plutôt un simple substitut du livre, de la méthode? Par exemple, l'application Mondly, ne transforme en rien la méthodologie. On est plutôt face à une méthodologie semblable à la méthodologie traditionnelle engoncée dans une enveloppe moderne. Ce qui revient à dire que l'apprentissage dans les langues étrangères ne dépendrait pas de l'outil, mais plutôt de la motivation du sujet apprenant, des facultés de l'enseignant pour motiver ses apprenants, et l'expérience préalable des uns et des autres avec la langue ou le pays, celle-ci étant peut-être la base de la motivation. Mais laissons pour l'instant de côté ces réflexions que nous reprendrons plus tard, pour aborder le dernier jeu ou game, c'est-à-dire le jeu vidéo et la vidéo interactive.

\subsection{Le jeu vidéo et la vidéo interactive}

À l'instar de la RM, peu de recherches pratiques ont été publiées sur le recours aux jeux vidéo et, surtout, aux vidéos interactives pour l'enseignement du (français) langue étrangère. Néanmoins, ces deux dernières années, on peut en trouver qui mettent en avant le lien entre les recherches réalisées dans d'autres langues et soulignent le côté interdisciplinaire et transversal de ce genre d'outils, par exemple chez Martínez et al. (2020), García-Pineda et al. (2020) ou encore Abal et al. (2020). De plus, ce dernier met en avant les bénéfices de la vidéo interactive dans le processus d'autoévaluation qui suppose un exercice d'autorégulation et d'autoréflexion critique en ce qui concerne le degré de connaissances des apprenants et le développement des compétences. La vidéo interactive, comme le jeu vidéo permettent ainsi de faciliter l'autoapprentissage et le développement de la réflexion critique et analytique (Sarmiento Bojórquez \& Cadena González, 2017).

La vidéo interactive, c'est une vidéo, donc un film qui se poursuit suivant les choix que l'on fait, comme dans Mondly. Il est vrai que la réalité du tournage est bien plus familière que ce que nous avons vu jusqu'à présent. Par contre, le degré d'immersion devrait être plus faible car il n'y a pas de manipulation d'objets virtuels, pas de casque qui plonge 
artificiellement le joueur dans une autre réalité. Malgré tout, le joueur-actant est immergé cognitivement et complètement. Il est pris par le jeu. Il s'agit d'immersion fictionnelle qui immerge complètement l'apprenant dans la narration (vidéo interactive) ou qui l'oblige à utiliser la langue pour pouvoir jouer, ce sont les jeux vidéo et jeux vidéo multi-joueurs. Ceux-ci permettent aux usagers de jouer à l'aide d'une console ou à l'aide d'un ordinateur. Dans ce dernier cas, il peut jouer à un jeu homme-machine ou à un jeu hommes-machine, c'est-à-dire le jeux vidéo multi-joueurs.

Dans les différents cas de jeux vidéo ${ }^{1}$ l'absence de casque provoquant l'immersion, rend celle-ci dépendante entièrement de l'apprenant qui peut ou pas s'immerger mentalement dans le jeu ou la narration et être "pris" par cette dernière, hypnotisé, et même devenir accros. Et entre les deux sortes de jeux vidéo, celui où l'interaction apparaît la plus naturelle, c'est quand l'on joue avec des joueurs aux quatre coins du globe. L'immersion, dans ce cas, est différente de celle que l'on a vu pour les différentes réalités virtuelles, augmentées et mixtes, ainsi que pour les jeux vidéo tout court ou la vidéo interactive. C'est l'exacte reproduction d'une immersion linguistique traditionnelle: le joueur, pour s'entendre avec un autre joueur, doit pouvoir parler et parle une langue étrangère à la sienne et ça fonctionne. C'est, il nous semble, jusqu'à présent et à notre connaissance, le moyen le plus efficace pour améliorer l'apprentissage d'une langue étrangère.

Tout cela conduit de nouveau à une réflexion autour des questions suivantes: la motivation et donc l'apprentissage dans les langues étrangères dépendent-elles d'une interaction quelconque, d'applications plus ou moins sophistiquées, de la nouveauté de l'expérience ou de l'objet, d'une immersion qui va de l'engagement à l'immersion totale, ou simplement de l'intérêt de résoudre un problème, d'une curiosité que l'on trouve dans le quotidien, dans la vie réelle ou dans une fiction, c'est-à-dire dans une histoire racontée, peu importe le support? Bien que tout cela ce ne soit pas les outils parfaits et idéaux, il ne faut pas que ces pistes de réflexion nous empêchent d'utiliser ces différentes applications et vidéos comme support des cours de langues étrangères. Le plus important, c'est l'implication de l'enseignant. Ce changement de perspective, de l'apprenant à l'enseignement, est indispensable car les deux sont en corrélation. Il est indispensable que l'enseignant prépare des balises afin de rassurer et de guider l'apprenant dans son apprentissage de cette langue étrangère imposée ou désirée, avec ces supports qui forcent une autre façon de faire. Ce qui implique d'aborder les soubassements de cette méthodologie $a d-h o c$ formative à l'aide d'un scénario ludoéducatif.

1 On peut citer comme jeu vidéo spécialement préparé pour l'apprentissage du français, le projet EVEIL de l'université de Strasbourg qui n'a malheureusement pas survécu et que nous n'avons pas pu utiliser. 


\section{Exemple de scénario ludoéducatif en classe de FLE avec un jeu vidéo}

\subsection{Définition}

Lorsque l'enseignant décide d'incorporer l'apprentissage immersif para technologie virtuelle dans son cours, l'objectif est que l'apprenant puisse apprendre en s'immergeant dans un monde alternatif, en devenant acteur et créateur et, si possible, en s'amusant.

Lors de la mise en place du scénario pédagogique, il faut faire cohabiter environnement virtuel et environnement d'apprentissage. Reprenant le scénario présenté par Schmoll (2017) ainsi que celui de Qotb (2015), il est nécessaire, dans un premier temps, de séparer les activités du cadre primaire (celle de l'environnement d'apprentissage, le contexte de classe) et les activités du cadre second (celle de l'environnement virtuel). Nous proposons ici un scénario adapté aux technologies virtuelles afin d'intégrer l'AI au sein de nos cours de langue:

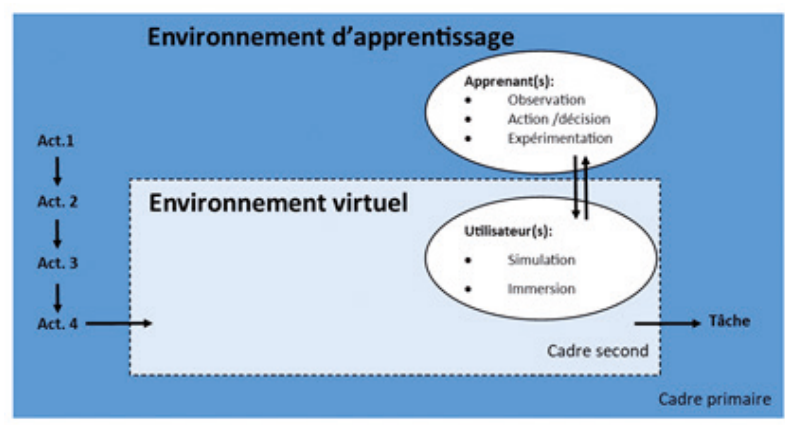

Figure 6: Modèle de scénario pour l'apprentissage immersif en classe (basé sur Schmoll, 2017 et Qotb, 2015).

Ainsi, nous avons un environnement d'apprentissage, que Schmoll (2017) nomme cadre primaire qui est mis en place bien avant l'immersion, autrement dit l'environnement virtuel ou cadre second. Soulignons cette relation réciproque entre les deux environnements dans la mesure ou les actions réalisées dans le monde réel, dans le cadre primaire, auront des répercussions directes sur celles réalisées dans le monde virtuel, le cadre second. L'utilisateur de l'environnement virtuel va agir en fonction de ses propres connaissances, acquises dans $1^{\prime}$ environnement d'apprentissage. Le scénario d'apprentissage du cadre primaire intègre ainsi des éléments de l'histoire du cadre second à travers des activités ou micro-tâches qui préparent les apprenants à ce dernier. Ce scénario sera donc composé de moments d'observation, de recherches, de prise de contact et de mise en commun et travaillera sur les contenus linguistiques et culturels essentiels pour mener à bien la mission du cadre second (Schmoll, 2017). Dans le cadre primaire, les apprenants vont découvrir le monde du cadre second et 
leur mission. La tâche finale présentées à la fin, lorsque l'apprenant revient dans le cadre primaire peut correspondre à un journal de bord revenant sur leur parcours, ou à une présentation revenant sur leur mission et les buts atteints.

\subsection{Exemples de scénarios}

Dans un mémoire de fin d'étude, l'étudiante Nieves Muelas Yunta (2018) propose un scénario ludique s'appuyant sur la méthodologie de l'apprentissage par le jeu. Elle propose l'utilisation en cours du jeu vidéo Soldats inconnus afin de travailler les temps du passé et de découvrir un aspect historique important: la première guerre mondiale. Soldats inconnus est un jeu d'aventure et de réflexion qui narre l'histoire commune de quatre personnages au milieu de la première guerre mondiale: Émile, un paysan de 50 ans combattant dans la guerre; Karl, un Allemand marié à la fille d'Émile et contraint à devenir ennemi de sa propre famille; Freddie, un Américain vivant à Paris; et Ana, une infirmière Belge qui rencontrera ces trois hommes. Au milieu, se trouve le chien Walt, un chien allemand de recherche de victimes, qui permettra de mettre en relation toutes ces histoires.
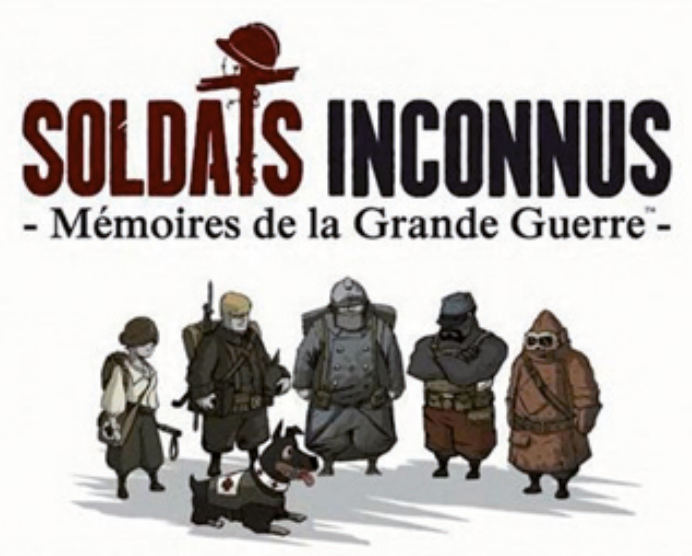

Figure 7: Image du jeu Soldats inconnus de Ubisoft Montpellier.

Le cadre primaire, ou environnement d'apprentissage, présente dans un premier temps le contexte de la première guerre mondiale et l'alternance des temps du passé dans un récit. L'enseignement devra présenter le contexte historico-social de façon simple et la grammaire devra être contextualisée à travers des textes et devra suivre une démarche inductive (observation réfléchie de la langue). L'enseignante présente l'environnement virtuel et la mission aux groupes d'apprenants, ainsi que les différents personnages du jeu. Dans le cadre second, le jeu vidéo entrera en jeu afin de permettre aux apprenants, par groupes de deux ou 
trois, de découvrir l'histoire de ces personnages. En fonction des choix réalisés, le chemin parcouru sera différent et ainsi chacun des groupes pourra par la suite présenter sa propre histoire vécue à travers le jeu.

L'exemple des Soldats inconnus de Ubisoft révèle qu'il est tout à fait possible de détourner un jeu vidéo à des fins qui vont au-delà de l'aspect ludique et qui servent l'apprentissage, et concrètement, celui des langues étrangères. Un autre support comme la réalité augmentée peut aussi aider à l'apprentissage d'une langue étrangère. Une expérience a été menée et décrite par Elena Vercher Ribis (2019) dans la revue Comunicación y pedagogía. Educación y tecnología. En conclusion, elle souligne que ce processus de Design Thinking a suscité de l'enthousiasme de la part des apprenants qui ont entre sept et douze ans. Dans ce cas-ci et contrairement au Soldat Inconnu, l'apprenant a fabriqué un jeu du type jeu de l'oie qui utilise la réalité augmentée. Pour ce faire, les enfants ont utilisé des applications et des robots comme HP Reveal et Bee boot.

Une autre expérience d'apprentissage immersif par technologie virtuelle a été mené par Papin (2019) à la faculté des sciences de l'éducation de l'université de Montréal. Dans sa thèse, il explique que le contexte, ou environnement d'apprentissage est celui du français québécois chez les étudiants internationaux qui voient les commerçants leur répondre en anglais plutôt qu'en français et souhaitent développer leur compétence en communication. Pour cela, il a mis en place un scénario de réalité virtuel (environnement virtuel) où les étudiants pouvaient simuler des situations de communication dans des lieux publics comme des bars, cafés, restaurants, etc. Les vidéos étant réelles, puisque filmées dans des endroits réels, l'expérience a été pertinente et efficace et a permis à ces étudiants de prendre confiance en eux et de développer aussi leur "désir de communiquer (DDC)" (Papin, 2019: 263). Néanmoins, le chercheur souligne l'impact de l'anxiété qu'une situation réelle provoque chez les étudiants (qu'il nomme anxiété situationnelle) qui n'a pas été aussi forte lors de cette expérience, ce qui montre que l'apprentissage immersif par technologies virtuelles peut apporter de grands bénéfices mais continue à avoir ses propres limites (Papin, 2019: 261). Les tâches réalisées ont toutes été contextualisées ou scénarisées dans une séquence pédagogique leur donnant sens. Papin (2019) explique ainsi:

Ainsi, l'idée de demander aux apprenants de réaliser plus d'une fois les simulations RV360, en intégrant celles-ci à une séquence didactique plus large, nous parait être un élément essentiel dans le but de développer la compétence communicative et le DDC (271).

Mais il y a bien d'autres réalités virtuelles que l'on peut mettre au service de l'un ou l'autre apprentissage. Parce qu'en effet, le but de l'apprentissage est primordial. Il ne suffit pas de vouloir utiliser une technologie immersive, il faut savoir pourquoi. Dans le cas de la langue étrangère, on sait que les stratégies sont variées, multiples, les compétences éga- 
lement, d'où l'intérêt du scénario pédagogique. Parallèlement, savoir quel outil est le plus approprié pour tel objectif est tout aussi important.

\section{Conclusion}

Les technologies immersives nous permettent d'enrichir nos cours de langues et aussi, et surtout, de proposer un cadre qui permettrait à l'apprenant de s'immerger complètement dans un monde alternatif à l'environnement d'apprentissage. Elles visent à mettre en situation et à transférer, du virtuel vers le réel, les connaissances et les compétences. Mais proposer une expérience d'AI ne doit pas se faire à la légère et un scénario pédagogique, proposant un cadre primaire qui préparerait les apprenants au cadre second, autrement dit à l'environnement virtuel, est nécessaire. De plus, l'enseignant doit s'assurer que la technologie soit assez riche afin de permettre aux apprenants de prendre des décisions différentes et de s'engager afin de mener à bien la mission et d'atteindre le but. Pour les langues étrangères, ce peut être un grand avantage, car la conception de tâche a grandement évolué depuis le Task Based Learning (TBL) et elle est orienté aujourd'hui vers des tâches non plus simulées, mais bien réelles. Pour ce qui concerne la réalité mixte, cette manipulation d'objet est justement réelle, en tout cas pour l'usager. Cependant, on peut se demander où est la frontière entre la pédagogie de projets dans une classe, qui, à notre avis, reste une simulation et l'agir hors de la classe? En outre, dans ce questionnement sur la simulation ou non de tâches, donc sur l'authenticité de ce que l'on fait ou ce que l'on ne fait pas, ne serait-ce pas, en fin de compte, la motivation ou le degré de motivation, et par là d'engagement de la personne qui est le principal élément pouvant faire varier l'apprentissage d'une langue étrangère? Cela nous amène à nous demander: est-ce que l'apprenant apprend mieux quand il utilise la RA, ou la RV, ou la RM? Et est-ce que cela ne dépend pas en fin de compte de l'apprenant même?

On peut reprendre notre idée de départ sur la remise en question de la réalité virtuelle en tant que technologie au service de l'apprentissage ou le serious game qui courent le danger de ne plus remplir les conditions d'un jeu et de se convertir en d'autres supports, au même titre que les livres ou méthodes, ce qui est appelé dégamification. Un exemple serait Assassin's Creed Discovery Tour face à Assassin's Creed.

Ce qui semble poser problèmes, c'est la relative passivité de l'apprenant, alors que c'est le contraire qui est recherché. En effet, l'apprenant, se retrouvant en classe, avec un outil "non-authentique", comme l'est le jeu sérieux, ou utilisant un outil "authentique" s'apparente à l'apprenant de l'approche communicative qui était passé d'un document fabriqué à un document authentique. Ce qui n'est pas négatif en soi, mais ce n'est pas ce que l'on poursuit car la passivité reste là. La nouveauté de la réalité à $360^{\circ}$, de la réalité virtuelle, de la réalité augmentée est bien sûr présente et c'est en ce qu'elle représente quelque chose de nouveau, qu'elle est motivante pour les apprenants. Mais, à notre avis, l'action n'est pas au rendez-vous. 
Anales de Filología Francesa, n. ${ }^{\circ}$ 29, 202

APPRENTISSAGE IMMERSIF DU FLE: POTENTIELS DES APPLICATIONS ET SCÉNARIO LUDO-ÉDUCATIF

Une possibilité pour ne pas tomber dans cet écueil, tout en gardant la prémices du CECRL, c'est-à-dire de faire de l'apprenant un être actif, ce qui faciliterait son apprentissage, c'est de faire en sorte que l'apprenant puisse ou bien s'immerger dans un jeu qui le motive et qu'il choisit, ou bien retourner la situation en faisant de l'apprenant un faiseur, un fabricant, un imagineur de réalité virtuelle. Et il le peut.

\section{Références bibliographiques}

Abal, A., Tanevitch, A., Pérez, P., González, A. \& M. Procopio Rodríguez. 2020. El video interactivo para la autoevaluación. III Jornadas sobre Las Prácticas Docentes. La Plata: <http://sedici.unlp.edu.ar/handle/10915/106083> [21/02/2021].

Arsenault, D., \& M. PiCARD. 2008. Le jeu vidéo entre dépendance et plaisir immersif: les trois formes d'immersion vidéoludique. Homo Ludens. Le jeu vidéo: un phénomène social massivement pratiqué. Congrès de l'ACTAS. doi: https://doi.org/10.3917/nre.011.0051

Fúchs, P., \& G. Moreau. 2003. Le traité de la réalité virtuelle, vol. 1. Paris, Les presses de l'École des Mines.

Garcia-Pineda, M., De Ves, E., Castaño, M., Roger, S., Cobos, M., Claver, J. \& J. GutierReZ-AgUADO. 2020. "Vídeos interactivos para mejorar el proceso enseñanza-aprendizaje en la generación YouTube" in Enseñanza Universitaria de la Informática, 353-356. Barcelona: AENUI: < http://www.aenui.net/ojs/index.php?journal=actas_jenui\&page=article \&op=view\&path $\% 5 \mathrm{~B} \% 5 \mathrm{D}=574 \&$ path $\% 5 \mathrm{~B} \% 5 \mathrm{D}=764>[21 / 03 / 2021]$.

Dugas, J. 2016. La réalité augmentée dans un contexte d'apprentissage: note de recherche. Thèse doctorale. Université de Rouen: <https://hal.archives-ouvertes.fr/hal-01349195> [12/03/2021].

Genvo, S. 2018. Du ludique au narratif. Enjeux narratologiques des jeux vidéo. Sciences du jeu, 9. doi:https://doi.org/10.4000/sdj.896.

Georges, F. 2013. L'immersion fictionnelle dans le jeu vidéo. Nouvelle revue d'esthétique, 1(11), 51-61: <https:/www.cairn.info/revue-nouvelle-revue-d-esthetique-2013-1-page-51. htm $>[17 / 04 / 2021]$.

Lewis, F., Plante, P. et Lemire, D. 2021. Pertinence, efficacité et principes pédagogiques de la réalité virtuelle et augmentée en contexte scolaire: une revue de littérature. Médiations Et médiatisations, (5), 11-27: <https://doi.org/10.52358/mm.vi5.161>[11/05/2021].

Marín-Díaz, V., \& B. E. Sampedro-Requena. 2020. "La Realidad Aumentada en Educación Primaria desde la visión de los estudiantes" in Alteridad, 15(1), 61-73: <https://doi. org/10.17163/alt.v15n1.2020.05> [21/04/2021].

Martínez Cortés, J., Guevara Bazán, I., Estrada Esquive, A., \& Encino Arambula, R. 2020. "El video interactivo: Recurso Innovador generador de Transversalidad en la Enseñanza" in EDUCATECONCIENCIA, 279-302: <http://tecnocientifica.com.mx/educateconciencia/index.php/revistaeducate/article/view/346/543> [18/05/2021].

Muelas Yuste, N. 2018. L'usage des TIC en classe de FLE: proposition didactique. Trabajo Fin de Grado: Universidad de Castilla La Mancha. 
Noeppel, G., Schlemminger, G., Roy, M., Veit, M., Capobianco, A. \& L. Schmoll. 2013. "Réalité virtuelle et jeux: de nouveaux outils pour des apprentissages plurilingues?" in Education et Sociétés(34), 29-42: doi:10.4000/esp.2731.

Ouramdane, N., Otmane, S. \& M. Mallem. 2009. “Interaction 3D en Réalité Virtuelle. État de l'art" in Techniques et sciences informatiques: doi: 10.3166/tsi.28.1017-1049.

Papin, K. 2019. La contribution des tâches de réalité virtuelle au désir de communiquer en français langue seconde à l'extérieur de la salle de classe à Montréal. Thèse doctorale. Université de Montreal.

ReINoso, R. 2019. "Realidad Aumentada y virtual en educación” in Comunicación y pedagogía. Educación y tecnología, 317-318.

Sarmiento Bojórquez, A. \& M. Cadena GonzÁlez. 2017. "Video Interactivo como Objeto de Aprendizaje en la Formación de los estudiantes de inglés en la Esc. Prep. Dr. Nazario V. Montejo Godoy" in IV Jornadas de TIC e Innovación en el Aula. La plata: Dirección de Educación a Distancia, Innovación en el aula y TIC (EAD): <http://sedici.unlp.edu.ar/handle/10915/65482> [21/02/2021].

Schmoll, L. 2017. "Penser l'intégration du jeu vidéo en classe de langue" in Recherche et pratiques pédagogiques en langues de spécialité, 36(2), 63-78: doi:https://doi.org/10.4000/ apliut.5722

Trepanier-Jobin, G., \& A. Couturier. 2018. "L'immersion fictionnelle au-delà de la narrativité" in Sciences du jeu, 9, 24-38: doi: https://doi.org/10.4000/sdj.950.

VideOlearning. "Définition: Apprentissage immersif (ou immersive Learning)" in Vidéo Learning Créateurs de compétences: <https://www.videolearning.fr/glossaire/apprentissageimmersif-immersive-learning/> [19/04/2021].

Wu, H. 2015. Les outils d'auto-organisation au sein d'une communauté d'apprentissage virtuelle. Grenoble: <https://dumas.ccsd.cnrs.fr/dumas-01224136> [12/05/2021]. 
